\title{
Optical sensing for stream flow observations: A review
}

\author{
Flavia Tauro, ${ }^{1}$ Andrea Petroselli, ${ }^{2}$ Salvatore Grimaldi ${ }^{1,3}$ \\ ${ }^{1}$ Department for Innovation in Biological, Agro-food and Forest Systems, University of Tuscia, Viterbo, Italy; \\ ${ }^{2}$ Department of Economics, Engineering, Society and Business Organisation, University of Tuscia, Viterbo, Italy; \\ ${ }^{3}$ Department of Mechanical and Aerospace Engineering, Tandon School of Engineering, New York University, \\ Brooklyn, NY, USA
}

\begin{abstract}
Images are revolutionising the way we sense and characterise the environment by offering higher spatial and temporal coverage in ungauged environments at competitive costs. In this review, we illustrate major image-based approaches that have been lately adopted within the hydrological research community. Although many among such methodologies have been developed some decades ago, recent efforts have been devoted to their transition from laboratories to operational outdoor settings. Sample applications of image-based techniques include flow discharge estimation in riverine environments, clogging dynamics in irrigation systems, and flow diagnostics in engineering infrastructures. The potential of such image-based approaches towards fully remote observations is also illustrated through a simple experiment with an unmanned aerial vehicle.
\end{abstract}

\section{Introduction}

Since the 1980s, high maintenance costs and limited resources have contributed to decrease the spatial coverage of hydrological

Correspondence: Salvatore Grimaldi, Department for Innovation in Biological, Agro-food and Forest Systems (DIBAF), University of Tuscia, via San Camillo De Lellis, Viterbo 01100, Italy.

E-mail: salvatore.grimaldi@unitus.it

Key words: Ungauged catchments; experimental monitoring; images; optical sensing; large scale particle image velocimetry; particle tracking velocimetry.

Acknowledgements: the authors thank Roberto Giannini, Massimiliano Lacquaniti, Giorgio Rossi, and Francesco Soffi for help with the experiments.

Received for publication: 22 January 2018.

Accepted for publication: 2 April 2018.

CCopyright F. Tauro et al., 2018

Licensee PAGEPress, Italy

Journal of Agricultural Engineering 2018; XLIX:836

doi:10.4081/jae.2018.836

This article is distributed under the terms of the Creative Commons Attribution Noncommercial License (by-nc 4.0) which permits any noncommercial use, distribution, and reproduction in any medium, provided the original author(s) and source are credited. observations and to prevent the implementation of dense monitoring networks in difficult-to-access environments and developing countries. In small hydrological catchments (less than $500 \mathrm{~km}^{2}$ ), hydrometric observations are typically lacking and collecting data may be unaffordable. To this end, empirical methods that do not require calibration procedures are applied, often leading to significant uncertainty and subjectivity.

Such criticalities have been highlighted by a recent survey conducted among 336 hydrologists (Blume et al., 2017). The results of the questionnaire pointed out the need for new measurement techniques and equipment, as well as for fostering field measurement and monitoring campaigns rather than for new modelling approaches. In this framework, in the latest years, a plethora of initiatives have blossomed to promote advances in hydrological measurements and environmental monitoring in general (Tauro et al., 2018). In the realm of hydrological monitoring, stream flow observations are essential for developing rating curves and calibrating rainfall-runoff models. Traditionally, experimental campaigns for stream flow measurements are rare and involve the deployment of expensive and bulky equipment in rivers. Thus, measurements are hardly ever taken in challenging conditions, such as during floods and in remote, small size streams with difficult topographies (Costa et al., 2000). Further, errors in water level and velocity observations are major sources of uncertainties in flow discharge estimations.Alternative to traditional stream flow measurement approaches, such as, for instance, current meters and acoustic Doppler current profilers, remarkable effort has been devoted to the development of non-contact flow sensing methodologies. Different from conventional sensors, images can be non-invasively captured and processed to characterise the flow current, thus minimising risk for operators and costly measurement equipment, see Figure 1. Also, with respect to alternative non-contact technologies, such as radars and coherent microwave systems, images offer higher spatial resolution at minimal costs. However, images are also highly sensitive to sunlight and illumination conditions and care must be taken in designing the experimental setup and in post-processing data to extract meaningful results. In latest years, the use of images has opened unprecedented capabilities to hydrologists by enriching their experimental toolbox with novel hardware and software approaches. In this review, we illustrate the major image-based techniques that have been developed in the research community. Further, we report a simple experiment on the use of images for fully remote surface flow velocity estimations.

\section{Image-based streamflow observations}

Images afford automated and nonintrusive observations. Their potential has been demonstrated in diverse realms of agricultural 
engineering, such as, the detection of dairy cows lying behaviour (Porto et al., 2013), the determination of the size distribution of wood chips (Febbi et al., 2013), the assessment of basal shear stress in debris-flow mixtures (D'Agostino et al., 2013), and the measurement of kinematic properties of granular flows (Gollin et al., 2015). With regards to fluid flow monitoring, digital images have been traditionally adopted in fluid dynamics laboratories as powerful quantitative fluid visualisation techniques. Here, we review the algorithms and procedures that are most commonly applied to monitor stream flow in hydrological systems.

\section{Large-scale particle image velocimetry}

Large-scale particle image velocimetry (LSPIV) applies the principles of the classical particle image velocimetry (PIV) technique to outdoor environments. PIV enables the estimation of the instantaneous flow velocity field of seeded fluids. A similar approach was utilised in Bettella et al. (2015) to measure the front velocity of small-scale debris flows. LSPIV was originally introduced by Fujita et al. (1997), whereby mappings of large-scale flows covering surfaces from 4 to $45,000 \mathrm{~m}^{2}$ were provided. The method was implemented on digital images of the free surface of laboratory and natural water bodies, and the experimental setup entailed a CCD camera oriented at an angle with respect to the water surface and buoyant tracing particles moving in the flow.

LSPIV is based on a high-speed cross-correlation scheme between an interrogation area (IA) in a first image (that is, an image taken earlier in time) and IAs within a search region (SR) in a second image (that is, an image taken at a time interval equal to the inverse of the camera acquisition frequency). To this end, each image is divided into a grid of IAs and the cross-correlation coefficients between IAs and SRs are computed. The location of the maximum value of the cross-correlation coefficient in consecutive frames yields displacement vectors, and can be determined at subpixel accuracy using fitting schemes. Based on the user-imposed camera acquisition frequency, it is then possible to estimate the instantaneous velocity from the displacement vectors. In laboratory settings, PIV parameters are set such that tracing particles move between consecutive images by at least a fourth of the IA. Also, a minimum of four to five particles should appear in each IA. However, the algorithm is independent on the presence of particleshaped objects in images. Specifically, it matches the distribution of gray-level intensities in digital frames and, therefore, it has been used to match flow patterns between successive images rather than individual tracers. To improve surface velocity estimation in noisy images, several modified cross-correlation schemes have been proposed for LSPIV. For instance, in Dobson et al. (2014), a temporal correlation-averaging algorithm has been introduced to merge correlation surfaces in the time domain and the scheme has been coupled with a signal-level indicator to remove noisy cross-correlations from the velocity computation. In Osorio-Cano et al. (2013), innovative image analysis encompassing image segmentation has been tested in laboratory conditions, and in Ran et al. (2016), the minimum quadratic difference algorithm was adopted to track surface patterns.

LSPIV-based flow velocity estimation entails the following phases: i) imaging of the field of view; ii) image ortho-rectification through transformation schemes that rely on the known coordinates of a minimum of six ground control points (GCPs) (in this phase, photometric calibration and camera lens distortion removal may also occur); and iii) image processing by high-speed crosscorrelation. In Tauro et al. (2014), an alternative setup has been proposed to prevent distortions from angled cameras and GCPs surveying. In this implementation, the camera optical axis is perpendicular to the water surface and medium-power lasers are placed at a known distance on the sides of the camera. Lasers create reference points in images at known distances, thus enabling fully remote photometric calibration. This setup generally leads to smaller fields of view where the resolution of both near and far
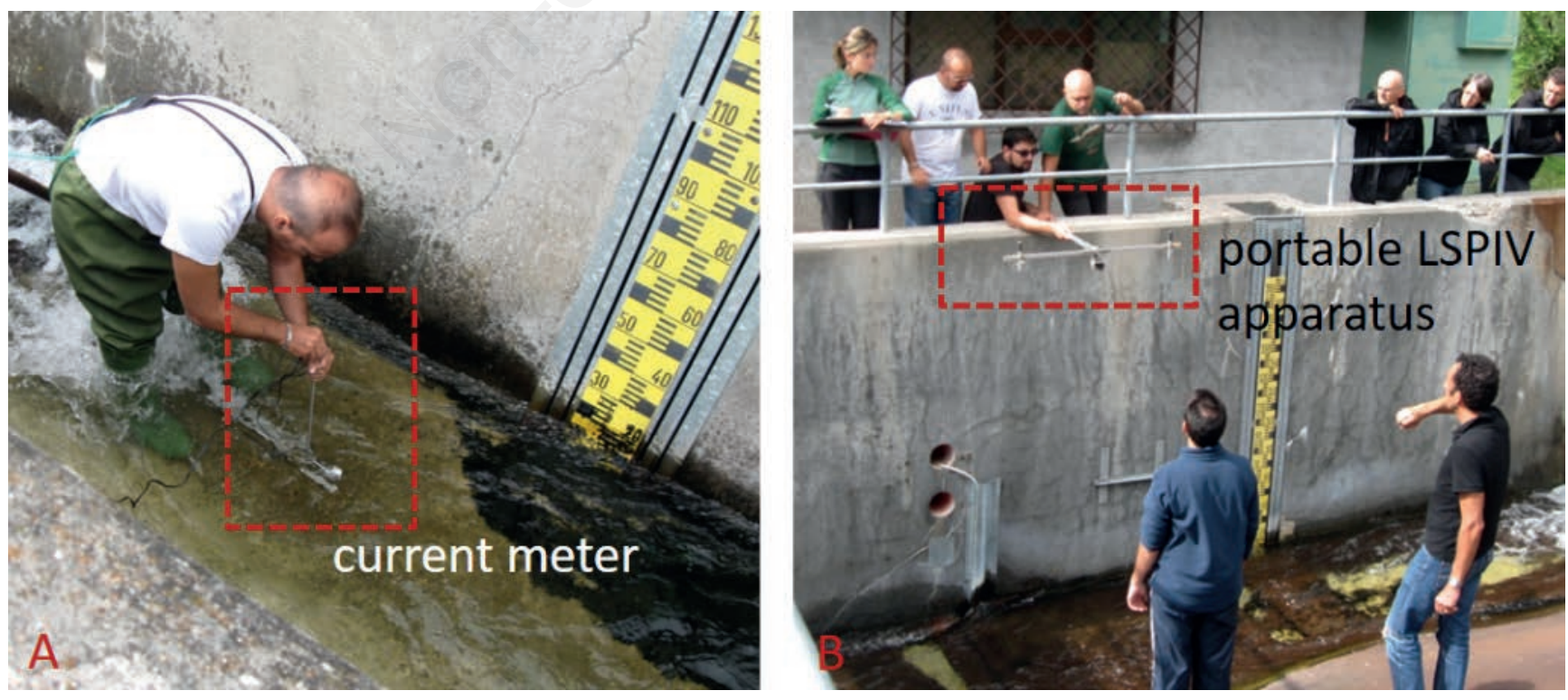

Figure 1. (A) Traditional vs (B) non-contact stream flow measurement approaches. In (A), an operator deploys a current meter in the Rio Cordon, Belluno, Italy. In (B), a portable apparatus is utilised to take pictures of the surface of the stream. 
field objects is acceptable. However, the use of lasers may be prevented in case of excessive sunlight and if the camera is rather distant (more than $20 \mathrm{~m}$ ) from the water surface. This alternative experimental configuration circumvents time-consuming GCPs surveying, which is sometimes impossible in difficult-to-access natural riverbanks, and only requires lens distortions removal.

The accuracy of this approach is largely dependent on the appearance of patterns on the water surface. LSPIV applications have utilised naturally occurring debris, foams, ice floes, waves, and boils as tracers. To this end, LSPIV implementations have been frequently installed downstream weirs and falls. However, the cross-correlation algorithm requires abundantly and continuously seeded surfaces and, therefore, tracers such as wood chips and leaves need to be manually added, see Figure 2. In case of slow, unseeded, and shallow flows, in Muste et al. (2005), the visibility of the water surface has been improved through waves generated by a fan. By recording images with and without waves and subtracting the velocity fields of both image data sets, the velocity of the water body has been obtained. In spite of remarkable efforts in surface seeding, the visibility of surface tracers is still an operating constraint to the technique, and it is typically enhanced through the use of filters, polarisers, or via image-based enhancement techniques. Although LSPIV has been widely adopted in natural environments, the absence of densely seeded surfaces has led to consistent flow velocity underestimations in diverse natural conditions (Tauro et al., 2014, 2015, 2016a, 2016b).

LSPIV has been demonstrated in a variety of experimental settings. In Hauet et al. (2008), a permanent LSPIV system was installed on the Iowa River to enable continuous discharge estimations for 23 months. To facilitate image processing, an adaptive IA approach was developed that allows adjusting the size of the IA according to image distortion and to scale the obtained velocity vectors. In Kim et al. (2008), a mobile LSPIV system was developed including a mast-mounted camera and a heavy-duty pick-up to enable rapid monitoring of riverine systems. These pioneering studies outlined illumination of the water surface, wind and rain effects, PIV parameterisation for variable flow conditions, and stage measurement as the key controls for accurate LSPIV-based flow discharge estimations. Also, through a numerical study and a vast number of experimental tests, the major sources of LSPIV measurement inaccuracy were attributed to surface seeding density, the acquisition of GCPs, and the process of image ortho-rectification.

Since the introduction of LSPIV, surface flow velocity measurements have been used to compute river discharge using standard velocity-area methods at selected cross-sections of known bathymetry. In Creutin et al. (2003), LSPIV-based discharge was found to be consistent with stage-discharge relationships, and the method was proposed as reliable to establish rating curves at natural sites. Alternatively to the velocity-area approach, in Bradley et al. (2002), LSPIV measurements were input to a hydraulic model and to derive three-dimensional flow fields for discharge estimation. In Hauet et al. (2009), LSPIV was complemented with photogrammetry to map waterway characteristics, including surface velocity, riverbank positions, flood plain edges, and erosion patterns, in riverine environments. LSPIV has also been advantageous to monitor high flow regimes that are typically not observable with costly and intrusive equipment. In Jodeau et al. (2008), surface flow velocities of a mountainous stream were measured during a reservoir release and then utilised to infer discharge through a sitespecific velocity coefficient. A mobile LSPIV device was also adopted to monitor surface velocity and estimate discharge during a fast greater than 10-year return period flood and a reservoir flushing release (Dramais et al., 2011). LSPIV was instrumental to esti- mate discharge in flash-flood conditions (LeCoz et al., 2010), whereby flow unsteadiness and image noise led to deviations ranging from $30-80 \%$. The uncertainty of LSPIV-based discharge estimations was further discussed in LeCoz et al. (2012), where the limitations of the velocity-area method were investigated.

Additional applications of LSPIV entailed flow diagnostics in engineering applications. For instance, in Kantoush et al. (2011), LSPIV was tested in several configurations, including river confluence and reservoirs during sediment flushing. Surface flow velocity measurements were instrumental to analyse the effects of basin geometry on the flow characteristics of reservoirs where large twodimensional coherent structures are prevalent. The influence of
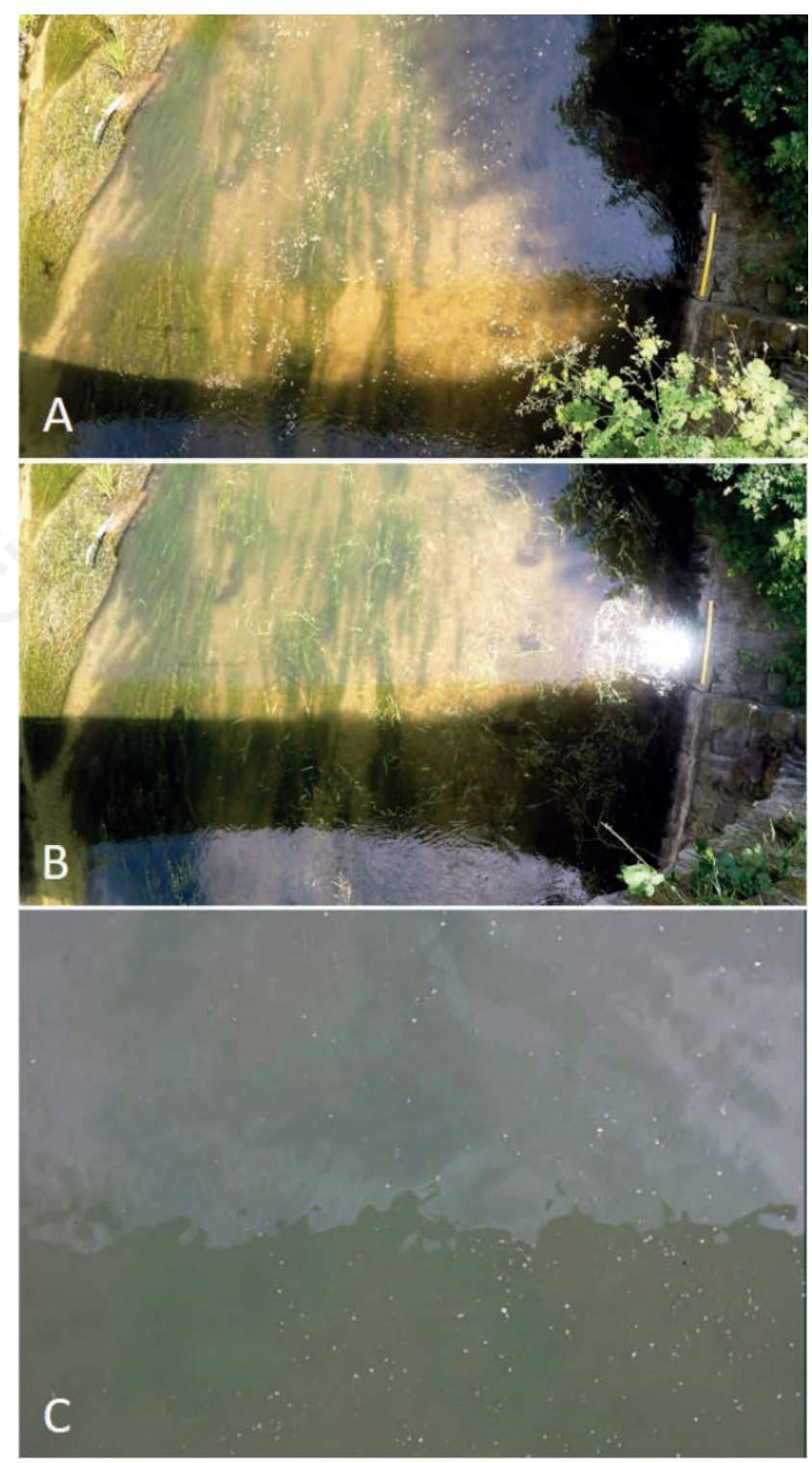

Figure 2. Appearance of tracer-seeded stream surfaces for largescale particle image velocimetry and/or particle-tracking velocimetry analyses. (A) and (B) display the surface of the Brenta river, Trento, Italy, seeded with wood chips and vegetation, respectively; $(C)$ depicts the surface of the Tevere river, Rome, Italy, as captured through the gauge-cam described in Tauro et al. (2017). 
reservoir geometry on sediment transport and deposition has been extensively studied with LSPIV. In particular, the technique proved effective in monitoring low velocity and shallow depth flow fields, which often challenge traditional instrumentation. Additionally, LSPIV was used to measure velocities during sediment flushing in reservoirs, where incisive channels are formed in the deposited material, thus controlling the time pattern of sediment released back into downstream rivers.

LSPIV has been highly beneficial for automatically investigating complex environments, where traditional approaches either fail or lead to inaccurate data. For instance, the technique was tested in large scale settings such as river estuaries, where tidal fluctuations and river in-flows can challenge measurements with alternative equipment. Also, the technique aided in characterising two-dimensional flow structures in a stream confluence. Shallow flows are also complex experimental settings that are typically difficult to survey with traditional measurement approaches and have been extensively studied with LSPIV. Additionally, LSPIV has been applied to characterise surface flows in vegetated rivers (Creëlle et al., 2018), small and irregular rivers (Gunawan et al., 2012), and mountain rivers (Stumpf et al., 2016). Advanced applications of LSPIV also entail the visualisation of particle motion in drip irrigation emitters to study and prevent clogging effects (Liu et al., 2016).

LSPIV low-cost equipment and the use of commercial RGB cameras for data generation support the diffusion of such a technique in environmental monitoring. Upon a short-time training, most operators are capable of taking adequate experimental videos, whereas post- processing phases such as image enhancement and processing should be executed by experts. In poorly gauged sites, the inherent simplicity of LSPIV acquisitions supported the use of crowd sourced footage and video data. In LeBoursicaud et al. (2015), a video of a flash flood taken from YouTube was analysed with LSPIV to estimate discharge. In LeCoz et al. (2016), citizen science projects in Argentina, France, and New Zealand entailed citizens capturing videos of floods and uploading them onto website platforms. The videos were then analysed with LSPIV and discharge was estimated (the bathymetry of the sites was surveyed after the events). Even if the accuracy of such measurements cannot be clearly estimated, these initiatives were preliminary attempts toward more dynamic and possibly wider measurement networks.

\section{Particle-tracking velocimetry}

Particle tracking velocimetry (PTV) consists of particle identification and tracking. In the first phase, images are processed to enhance the appearance of particles in the field of view (for instance, by applying filters and thresholds) and the location of the centroid of the particles in frames is recovered. In the tracking phase, the centroid of the detected particles is identified in subsequent images to reconstruct particle trajectory. Several algorithms have been developed for PTV analysis. In Brevis et al. (2011), cross-correlation is implemented for both particle detection and tracking. In addition, relaxation, heuristics based on a priori knowledge of the flow, and Voronoï tracking scheme have been utilised.

PTV is designed for low seeding density flows and does not require assumptions on flow steadiness nor on the relative position of neighbour particles. Its applications in the literature are diverse and involve dispersion in porous media (Moroni and Cushman, 2001), exchange processes between rivers and groynes (Yossef and deVriend, 2011), and tidal patterns (Kimura et al., 2011). Since PTV is dependent on the presence of round-shaped tracers, its use for stream flow observations is less frequent than LSPIV, which may be applied also without deploying objects in the current.

Advantages and disadvantages of LSPIV and PTV are reported in Table 1. The experimental setup required for PTV is consistent with LSPIV and relies on inexpensive cameras and, eventually, lasers for remote calibration. If cameras are angled to the water surface, similar to LSPIV, image pre-processing should include ortho-rectification. On the other hand, different from LSPIV, PTV typically mandates particles to be highly defined and is most commonly implemented with round-shaped floaters in case of in situ studies. For a comparison between LSPIV and PTV, refer to Tauro et al. (2017). Upon PTV processing, particle trajectories are reconstructed based on velocity vectors that are randomly located in the field of view. Surface flow velocity maps can be generated by interpolating particle trajectories.

\section{Alternative approaches}

In addition to LSPIV and PTV, numerous image-based approaches for flow observations have been developed in the last decade. Most of them aim at extracting flow velocity based on the transit of visible features in the field of view. For instance, the space-time image velocimetry technique assumes that the brightness distribution in images is dependent on surface velocity (Fujita

Table 1. Experimental details and advantages and disadvantages of large-scale particle image velocimetry and particle tracking velocimetry for hydrological applications.

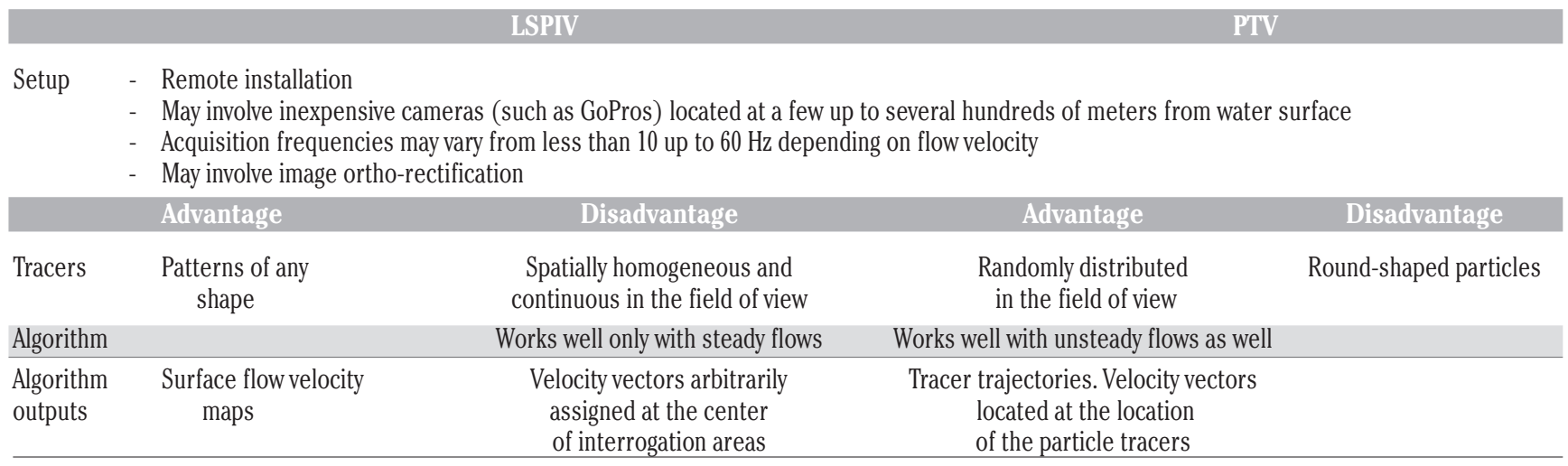

LSPIV, large-scale particle image velocimetry; PTV, particle-tracking velocimetry. 
et al., 2007). The approach is utilised to measure the orientation angle of patterns in images and leads to stream wise velocity distribution. This methodology is sensitive to noise and some algorithms have been recently introduced to improve velocity estimation. The appearance of surface features is crucial for accurately estimating surface flow velocity. For instance, both LSPIV and PTV are negatively impacted by excessive illumination and low acquisition frequency. To this end, several alternative approaches have been proposed. In Tauro et al., 2011, a cross-correlation based PTV algorithm was proposed and combined with highly visible tracers to recover the trajectory of severely deformed tracers due to low shutter speeds. The approach has been tested in several settings, including a mountainous stream. In Perks et al. (2016), the Kanade-Lucas-Tomasi algorithm was applied to track surface features during a flood event. This optical flow algorithm is independent on the shape of the tracers and less computationally expensive than cross-correlation. However, its use in hydrology is limited to a few applications in rainfall monitoring and to proof-of-concept experiments in river and ocean settings.

\section{Integration of images and unmanned aerial system technology}

The presented image-based methodologies are frequently implemented at permanent gauging stations (which are often located nearby existing monitoring units) and through portable setups (simple hand-held masts or mobile equipment integrated onto trucks). Latest research has entailed the challenging combination of image analysis and unmanned aerial vehicle (UAV) technology. In agricultural engineering, UAVs are bridging the gap between traditional aerial and satellite imagery and ground-based monitoring toward more accurate and highly resolved geospatial data. For instance, UAVs are expected to open novel avenues in the exploration of calanchi erosion and landform evolution (Caraballo-Arias and Ferro, 2016).

The integration of images and manned aerial surveys dates back to more than a decade ago. The pioneering work by Fujita and Hino (2003) demonstrated LSPIV on image sequences captured from a helicopter in low flow and flood conditions. In Fujita and Kunita (2011), aerial images of the 2002 flood of the Yodo River were processed with LSPIV to reconstruct surface flow velocity maps.

The advent of UAVs has enabled flow observations in difficultto-access environments at low costs and with minimal equipment. In fact, commercial UAVs offer good stability and controllability at competitive prices. They can be easily retrofitted with sport cameras, such as GoPros, which enable high-frequency and high-resolution footage. Experimental tests are simple and can be executed by users after minimal training. Specifically, the UAV can be navigated above the water surface of interest and then station kept above it to record stable videos of the flow. The platforms can be hovered for a few minutes at acquisition frequencies ranging from 15 to $30 \mathrm{~Hz}$ to gather video sequences of hundreds of images. The acquisition frequency should be tuned based on the water flow velocity. Buoyant tracers may be deployed onto the surface depending on the appearance of the water and on the adopted algorithm. Image post-processing is consistent with conventional ground-based image approaches: before applying velocimetry algorithms, frames are enhanced and ortho-rectified if the camera axis is angled to the surface. Due to UAV vibrations, image matching may be necessary to analyse consistent fields of view in consecutive images. However, due to strong winds, inaccurate station keeping may hamper image-based mea- surements. Commercial UAVs offer limited hovering capabilities (for instance, the DJI Phantom2 has a vertical hovering accuracy of $0.8 \mathrm{~m}$ and a horizontal accuracy of $2.5 \mathrm{~m}$ according to: http://www.dji.com/). In Tauro et al. (2016b), an hovering assessment conducted with a DJI Phantom 2 demonstrated that images taken during light air wind conditions $(2.19 \mathrm{~km} / \mathrm{h}$ wind speed $)$ displayed minimal changes and were, therefore, acceptable for stream flow measurements. Finally, photometric calibration is performed by capturing objects of known dimensions in the field of view (such as, for instance, the river width) or by using laser pointers mounted onboard the platform.

In Tauro et al. (2015), a simple UAV platform was built and deployed to capture video data processed with LSPIV. In Tauro et al. (2016a, 2016b), an off-the-shelf UAV platform was also characterised to assess its suitability to capture image data and then used to estimate surface flow velocity in a mountainous stream. Flow estimations were in close agreement with benchmark data and images offered improved quality with respect to traditional ground-based LSPIV implementations. Similar experiments were described by other authors (Perks et al., 2016).

Interestingly, besides stream flow observations, UAVs are offering a variety of inexpensive data at sites that may be impossible to survey with traditional sensing instrumentation. For instance, recent advances enable the acquisition of water level, surface-groundwater interaction, and bathymetry. A limitation to the pervasive use of UAVs in environmental monitoring practice lays in current regulations to safely navigate such devices. Currently, most countries require licensed pilots and only allow line-of-sight navigation. However, continuous progress in the autonomy of UAVs is expected to facilitate their use in science and engineering.

\section{Fully remote stream flow measurements in an ungauged stream reach}

Here, we present a proof-of-concept experiment on the integrated use of UAV technology and image analysis for surface flow observations in an ungauged stream reach. The experiment was executed on the Marta river, a few hundred meters downstream the Bolsena lake, in September 2016. Both the LSPIV and PTV algorithms were applied to estimate surface flow velocity.

\section{Experimental site}

The Marta river is the emissary of the Bolsena lake, Viterbo, Italy. Its hydrological catchment has an area of $1090 \mathrm{~km}^{2}$ and a total length of $60 \mathrm{~km}$. The river has no tributaries for the initial 20 $\mathrm{km}$, where its flow discharge is largely regulated by the Bolsena lake. Two water gauge stations are located along the river. According to the station located at Ponte della Cartiera $(13 \mathrm{~km}$ downstream the Bolsena lake), the average annual discharge is $2.42 \mathrm{~m}^{3} / \mathrm{s}$. Close to the Bolsena lake, the river has an average width of less than $10 \mathrm{~m}$ and a depth of $0.5 \mathrm{~m}$.

\section{Unmanned aerial vehicle platform and image processing}

A low-cost DJI F550 hexacopter was assembled and retrofitted with six E310 brushless motors $(960 \mathrm{rpm} / \mathrm{V}), 3 \mathrm{~S} 5200 \mathrm{mAh}$ full power LiPO batteries, and a DJI Naza-M V2 control board. Videos were taken with a GoPro Hero 4 Black edition, mounted on a twoaxis gimbal and with its axis perpendicular to the water surface to circumvent image ortho-rectification. Data were gathered by manually taking off the UAV and then navigating it to the centre of the river at less than $10 \mathrm{~m}$ above the water surface, see the sketch in 
Figure 3. Then, the UAV was flown in the hovering mode (the device stability is continuously corrected via satellite signals). To enhance flow appearance, wood chips ( 1 to $5 \mathrm{~cm}$ in diameter) were manually deployed onto the water surface as buoyant tracers. The tracers were homogeneously and continuously deployed onto the surface to facilitate image processing through LSPIV. The GoPro captured videos at $30 \mathrm{~Hz}$ acquisition frequency and full $\mathrm{HD}$ resolution $(1920 \times 1080$ pixels $)$. Here, we report surface flow velocity estimations obtained by analysing a sequence of 101 consecutive frames $(3.4 \mathrm{~s})$. The sequence was selected based on tracer homogeneity in the field of view.

The image sequence was processed according to the workflow in Figure 3: i) frames were converted to grayscale and lens distortion corrected; ii) images were then stabilised to prevent the UAV vibrations to yield slight changes in the field of view; iii) photometric calibration was executed to assign metric dimensions to image pixels; and iv) the sequence was processed through LSPIV and PTV using the Matlab toolboxes PIVlab (Thielicke and Stamhuis, 2014) and PTVlab (Brevis et al., 2011), respectively. Even if UAV vibrations were demonstrated to be acceptable for surface flow observations (Tauro et al., 2016b), the frame sequence was stabilised to reduce eventual inaccuracies in velocity estimations. To this end, a custom-built Matlab-based procedure was developed that detects relevant features in images by applying the FAST algorithm, and then maps consistent features in consecutive frames through the RANSAC approach. After vibration removal, consecutive frames exhibited a mean squared error of approximately 70 pixel $^{2}$ as compared to an average value of 150 pixel $^{2}$ before processing. The vibration removal correction aimed at selecting consistent fields of view in consecutive images within the video sequence. To this end, the procedure rotated and translated the images, thus eliminating the effects due to the UAV-mounted camera. Photometric calibration was remotely executed by automatically extracting the river cross-section width from images and then relating such a distance in pixels to the metric width of the river as measured from Google Earth $(7.57 \mathrm{~m}$ at the experimental site). The automated extraction of the pixel width of the river was performed by developing a custom-built procedure that enhances contrast differences between the water surface and the river width, and then applies Otsu's segmentation approach to
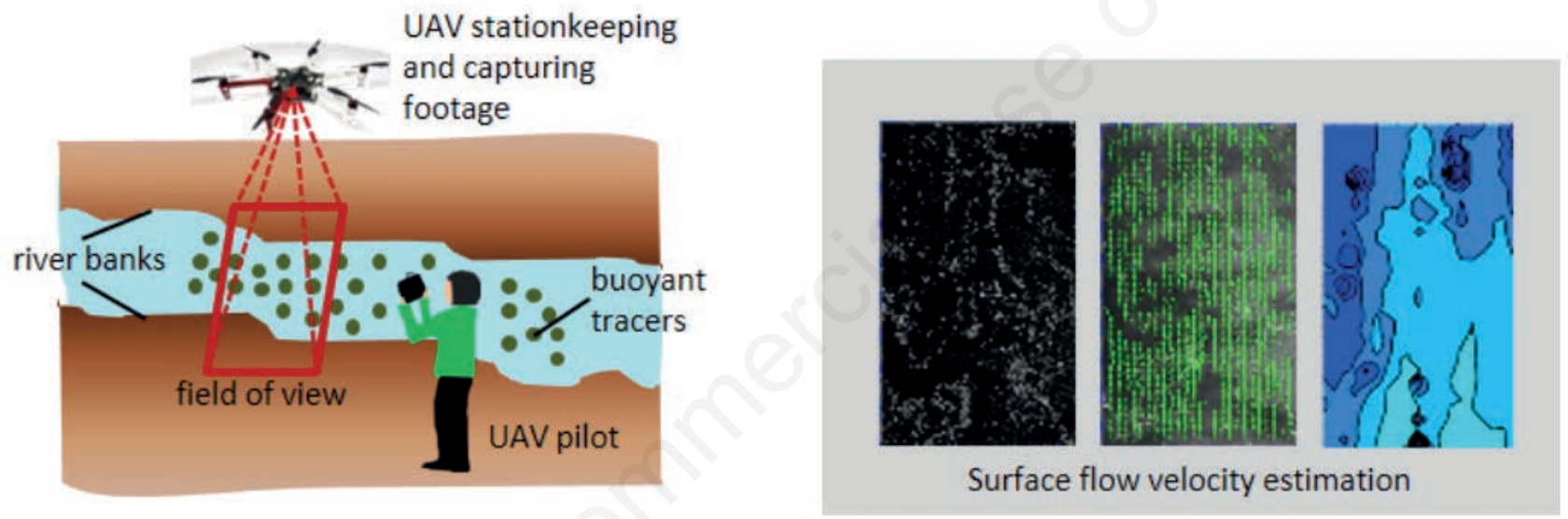

Figure 3. Sketch of the proof-of-concept experiment performed with the DJI F550 on the Marta river, Viterbo, Italy (left panel); image processing from raw images to velocity maps (right panel). UAV, unmanned aerial vehicle.

A

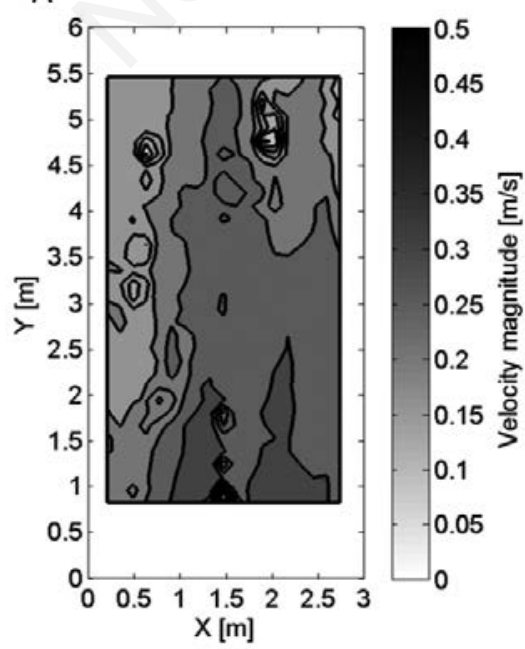

B

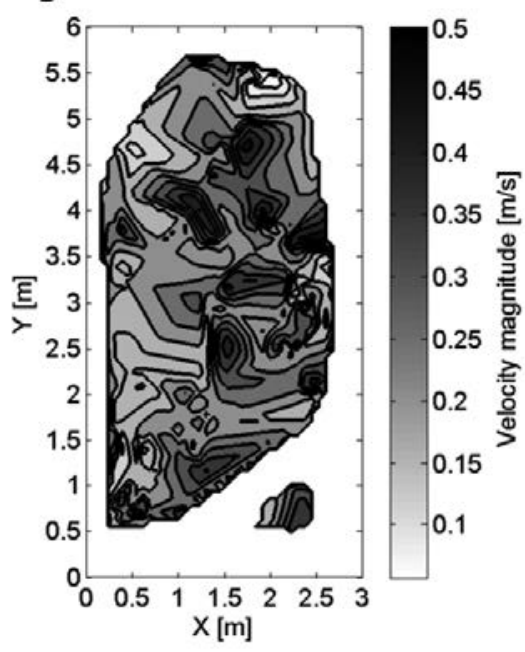

Figure 4. (A) Time-averaged surface flow velocity map of the Marta river obtained through large-scale particle image velocimetry and (B) through particle-tracking velocimetry. 
identify the pixels pertaining to water. This fully remote approach was in line with the calibration conducted by taking pictures of a meter stick in the field of view.

To execute LSPIV and PTV analyses, a region of interest depicting the densest amount of tracers in the field of view was selected in frames. PTV analyses were performed by preliminarily enhancing the appearance of the tracers by subtracting the mean grayscale intensity from images. Then, particle detection was executed through cross-correlation with a Gaussian kernel (intensity grayscale level set to 80 , standard deviation to 7 pixels, and correlation threshold to 0.5 ). Particle tracking was achieved by crosscorrelation (interrogation window set to 15 pixels, minimum correlation threshold to 0.5 , and similarity among neighbour particles to $20 \%$ ). Upon PTV analysis, a surface flow velocity map was interpolated from particle trajectories.

For PIV analysis, a region of interest consistent to PTV was selected, and image quality was enhanced through a Gaussian high pass filter (10 pixels in size). Direct cross-correlation was applied on the sequence of images by setting the interrogation window size to $32 \times 32$ pixels and the grid size to $16 \times 16$ pixels. The $2 \times 3$-point Gaussian fit was used as sub-pixel displacement peak estimator. Data were validated by applying velocity upper and lower thresholds. Thresholds were determined as $\bar{u} \pm n^{*} \sigma_{\mathrm{u}}$, with $\bar{u}$ the mean velocity for each analysed frame pair, $\sigma_{u}$ its standard deviation, and $\mathrm{n}$ set to 1.5 . Such a parameter was selected by assuming a Gaussian distribution of measurement errors due to irregular changes in the appearance of the water surface from light reflections. The velocity field for the frame sequence was obtained by averaging in time the velocity field computed for each frame pair.

\section{Remote surface flow velocity estimation}

Figure 4 displays PIV (A) and PTV (B) time-averaged surface flow velocity maps for the analysed sequence. By computing the average velocity of the map, PIV-based velocity was equal to 0.29 $\mathrm{m} / \mathrm{s}$ with standard deviation equal to $0.055 \mathrm{~m} / \mathrm{s}$. PTV-based velocity was instead equal to $0.31 \mathrm{~m} / \mathrm{s}$ with a standard deviation of 0.071 $\mathrm{m} / \mathrm{s}$. PTV velocities presented slightly positive skewness, whereas the skewness of PIV data was negative and, therefore, velocities smaller than the average were highly frequent. The PTV-based map displays empty regions since values were interpolated only in areas where the transit of particle tracers was observed. The appearance of the maps is quite different since PIV captures the average behaviour of the flow, whereas PTV is influenced by the transit of individual particles.

Overall, both techniques were in close agreement and showed potential to remotely estimate surface flow velocity in the ungauged experimental site. Remarkably, the experiment was conducted in less than two hours by three operators. Further, costs of the tracer materials were minimal and the cost of the platform is less than $1000 €$. Flow discharge estimations may be roughly obtained by combining information on surface velocity with the bathymetry of the stream reach. This simple experiment demonstrates the simplicity of image-based approaches for environmental monitoring and their transformative impact for hydrological and agricultural applications.

\section{Conclusions and future perspectives}

In this review, we illustrated latest advances on optical sensing approaches for stream flow observations. These relatively new image-based approaches demonstrate potential towards low-cost and rapid surveys of difficult-to-access and ungauged areas, thus offering a viable alternative to costly and spatially-scattered traditional measurement stations. Although many of the presented methodologies have been introduced several years ago, imagebased approaches still need a thorough assessment to be regularly adopted in monitoring practice. Building novel sensing instrumentation requires extensive calibration and validation, and sometimes collaboration from local authorities to install novel equipment in natural systems or at existing gauging stations. Future perspectives on the use of optical sensing for environmental monitoring are hardly imaginable. Technological advances have accustomed us to extremely high quality videos at high speed frequency in the span of a few years. In this framework, we expect that continuous technological progress may sensibly empower the capabilities and cost-effectiveness of current optical sensors, thus shedding new light on our comprehension of natural ecosystems. Thermal imagery is most probably set to be at the forefront of environmental monitoring, by enabling less noisy acquisitions independently on illumination conditions. Currently, thermal images have leveraged many applications in agricultural engineering, such as, for instance, flow observations in streams (Tauro and Grimaldi, 2017) and investigations on the resilience of plants to stress (Ludovisi et al., 2017). In the near future, we expect thermal cameras to become powerful yet affordable diagnostic instruments to rapidly and thoroughly unveil complex stream flow physics.

\section{References}

Bettella F, Bischetti GB, D’Agostino V, Marai SV, Ferrari E, Michelini T, 2015. Comparison of measurement methods of the front velocity of small-scale debris flows. J. Agr. Eng. 46:472.

Blume T, vanMeerveld I, Weiler M, 2017. The role of experimental work in hydrological sciences - insights from a community survey. Hydrolog. Sci. J. 62:334-7.

Bradley AA, Kruger A, Meselhe EA, Muste MVI, 2002. Flow measurement in streams using video imagery. Water Resour. Res. 38:1-8.

Brevis W, Niño Y, Jirka GH, 2011. Integrating cross-correlation and relaxation algorithms for particle tracking velocimetry. Exp. Fluids 50:135-47.

Caraballo-Arias NA, Ferro V, 2016. Assessing, measuring and modelling erosion in Calanchi areas: a review. J. Agr. Eng. 47:573.

Creëlle S, Roldan R, Herremans A, Meire D, Buis K, Meire P, Van Oyen T, De Mulder T, Troch P, 2018. Validation of large-scale particle image velocimetry to acquire free-surface flow fields in vegetated rivers. J. Appl. Water Eng. Res. 6:171-82.

Creutin JD, Muste M, Bradley AA, Kim SC, Kruger A, 2003. River gauging using PIV techniques: a proof of concept experiment on the Iowa River. J. Hydrol. 277:182-94.

D'Agostino V, Bettella F, Cesca M, 2013. Basal shear stress of debris flow in the runout phase. Geomorphology 201:272-80.

Dobson DW, Holland KT, Calantoni J, 2014. Fast, large-scale, particle image velocimetry- based estimation of river surface velocity. Comput. Geosci. 70:35-43.

Dramais G, LeCoz J, Camenen B, Hauet A, 2011. Advantages of a mobile LSPIV method for measuring flood discharges and improving stage-discharge curves. J. Hydro-Environ. Res. 5:301-12

Febbi P, Costa C, Menesatti P, Pari L, 2013. Determining wood 
chip size: image analysis and clustering methods. J. Agr. Eng. 44(s2):e102.

Fujita I, Hino T, 2003. Unseeded and seeded PIV measurements of river flows video from a helicopter. J. Visual.-Japan 6:245-52.

Fujita I, Kunita Y, 2011. Application of aerial LSPIV to the 2002 flood of the Yodo River using a helicopter mounted high density video camera. J. Hydro-Environ. Res. 5:323-31.

Fujita I, Muste M, Kruger A, 1997. Large-scale particle image velocimetry for flow analysis in hydraulic engineering applications. J. Hydraul. Res. 36:397-414.

Fujita I, Watanabe H, Tsubaki R. 2007. Development of a nonintrusive and efficient flow monitoring technique: the spacetime image velocimetry (STIV). Int. J. River Basin Manag. 5:105-14.

Gollin D, Bowman E, Shepley P, 2015. Methods for the physical measurement of collisional particle flows. IOP Conf. Series: Earth and Environmental Science, 26:012017.

Gunawan B, Sun X, Sterling M, Shiono K, Tsubaki R, Rameshwaran P, Knight DW, Chandler JH, Tang X, Fujita I, 2012. The application of LS-PIV to a small irregular river for inbank and overbank flows. Flow Meas. Instrum. 24:1-12.

Hauet A, Kruger A, Krajewski W, Bradley A, Muste M, Creutin J, Wilson M, 2008. Experimental system for real-time discharge estimation using an image-based method. J. Hydrol. Eng. 13:105-10.

Hauet A, Muste M, Ho H-C, 2009. Digital mapping of riverine waterway hydrodynamic and geomorphic features. Earth Surf. Proc. Land. 34:242-52.

Jodeau M, Hauet A, Paquier A, Le Coz J, Dramais G, 2008. Application and evaluation of LS-PIV technique for the monitoring of river surface velocities in high flow conditions. Flow Meas. Instrum. 19:117-27.

Kantoush SA, Schleiss AJ, Sumi T, Murasaki M, 2011. LSPIV implementation for environmental flow in various laboratory and field cases. J. Hydro-Environ. Res. 5:263-76.

Kim Y, Muste M, Hauet A, Krajewski WF, Kruger A, Bradley A, 2008. Stream discharge using mobile large-scale particle image velocimetry: A proof of concept. Water Resour. Res. 44:W09502.

Kimura N, Liu W-C, Wu CH, Bechle AJ, Chen W-B, Huang W-C, 2011. Flow measurement with multi-instrumentation in a tidalaffected river. Water Environ. J. 25:563-72.

LeBoursicaud R, Pénard L, Hauet A, Thollet F, LeCoz J, 2015. Gauging extreme floods on YouTube: application of LSPIV to home movies for the post-event determination of stream discharges. Hydrol. Proc 30:90-105.

LeCoz J, Hauet A, Pierrefeu G, Dramais G, Camenen B, 2010. Performance of image-based velocimetry LSPIV applied to flash-flood discharge measurements in Mediterranean rivers. J. Hydrol. 394:42-52.

LeCoz J, Camenen B, Peyrard X, Dramais G, 2012. Uncertainty in open-channel discharges measured with the velocity-area method. Flow Meas. Instrum. 26:18-29.

LeCoz J, Patalano A, Collins D, Guillén NF, García CM, Smart GM, Bind J, Chiaverini A, LeBoursicaud R, Dramais G, Braud I, 2016. Crowdsourced data for flood hydrology: feedback from recent citizen science projects in Argentina, France, and New Zealand. J. Hydrol. 541:766-77.

Liu H, Sun H, Li Y, Feng J, Song P, Zhang M, 2016. Visualizing particle movement in flat drip irrigation emitters with digital particle image velocimetry. Irrig. Drain. 65:390-403.
Ludovisi R, Tauro F, Salvati R, Khoury S, Scarascia Mugnozza G, Harfouche A, 2017. UAV-based thermal imaging for highthroughput field phenotyping of black poplar response to drought. Front. Plant Sci. 8:1681.

Moroni M, Cushman JH, 2001. Three-dimensional particle tracking velocimetry studies of the transition from pore dispersion to Fickian dispersion for homogeneous porous media. Water Resour. Res. 37:873-84.

Muste M, Schöne J, Creutin J, 2005. Measurement of free-surface flow velocity using controlled surface waves. Flow Meas. Instrum. 16:47-55.

Osorio-Cano JD, Osorio AF, Medina R, 2013. A method for extracting surface flow velocities and discharge volumes from video images in laboratory. Flow Meas. Instrum. 33:188-96.

Perks MT, Russell AJ, Large ARG, 2016. Technical Note: Advances in flash flood monitoring using unmanned aerial vehicles (UAVs). Hydrol. Earth Syst. Sci. 20:4005-15.

Porto SMC, Arcidiacono C, Anguzza U, Giummarra A, Cascone G, 2013. An automatic system for the detection of dairy cows lying behaviour in free-stall barns. J. Agr. Eng. 44(s2):e31.

Ran QH, Li W, Liao Q, Tang H-L, Wang M-Y, 2016. Application of an automated LSPIV system in a mountainous stream for continuous flood flow measurements. Hydrol. Proc. 30:3014-29.

Stumpf A, Augereau E, Delacourt C, Bonnier J, 2016. Photogrammetric discharge monitoring of small tropical mountain rivers: a case study at Rivière des Pluies, Réunion Island. Water Resour. Res. 52:4550-70.

Tauro F, Grimaldi S, 2017. Ice dices for monitoring stream surface velocity. J. Hydro-Environ. Res. 14:143-9.

Tauro F, Pagano C, Phamduy P, Grimaldi S, Porfiri M, 2015. Large scale particle image velocimetry from an unmanned aerial vehicle. IEEE/ASME T. Mech. 20:3269-75.

Tauro F, Pagano C, Porfiri M, Grimaldi S, 2011. Tracing of shallow water flows through buoyant fluorescent particles. Flow Meas. Instrum. 26:93-101.

Tauro F, Petroselli A, Arcangeletti E, 2016a. Assessment of dronebased surface flow observations. Hydrol. Proc. 30:1114-30.

Tauro F, Porfiri M, Grimaldi S, 2014. Orienting the camera and firing lasers to enhance large scale particle image velocimetry for streamflow monitoring. Water Resour. Res. 50:7470-83.

Tauro F, Porfiri M, Grimaldi S, 2016b. Surface flow measurements from drones. J. Hydrol. 540:240-5.

Tauro F, Piscopia R, Grimaldi S, 2017. Streamflow observations from cameras: large-scale particle image velocimetry or particle tracking velocimetry? Water Resour. Res. 53:10374-94.

Tauro F, Selker J, vandeGiesen N, Abrate T, Uijlenhoet R, Porfiri M, Manfreda S, Caylor K, Moramarco T, Benveniste J, Ciraolo G, Estes L, Domeneghetti A, Perks MT, Corbari C, Rabiei E, Ravazzani G, Bogena H, Harfouche A, Brocca L, Maltese A, Wickert A, Tarpanelli A, Good S, LopezAlcala JM, Petroselli A, Cudennec C, Blume T, Hut R, Grimaldi S, 2018. Measurements and Observations in the XXI century (MOXXI): innovation and multidisciplinarity to sense the hydrological cycle. Hydrol. Sci. J. 63:169-96.

Thielicke W, Stamhuis EJ, 2014. PIVlab - Towards user-friendly, affordable and accurate digital particle image velocimetry in MATLAB. J. Open Res. Soft. 2:1-10.

Yossef MFM, deVriend HJ, 2011. Flow details near river groynes: experimental investigation. J. Hydraul. Eng. 137:504-16. 